\title{
Nanoscopic quantification of sub-mitochondrial morphology, mitophagy and mitochondrial dynamics in living cells derived from patients with mitochondrial diseases
}

Weiwei Zou ${ }^{1,2 \dagger}$, Qixin Chen ${ }^{3,4+}$, Jesse Slone ${ }^{1,5}$, Li Yang ${ }^{1,6}$, Xiaoting Lou ${ }^{1,7}$, Jiajie Diao ${ }^{3^{*}}$ and Taosheng Huang ${ }^{1,5^{*}}$

\begin{abstract}
SLC25A46 mutations have been found to lead to mitochondrial hyper-fusion and reduced mitochondrial respiratory function, which results in optic atrophy, cerebellar atrophy, and other clinical symptoms of mitochondrial disease. However, it is generally believed that mitochondrial fusion is attributable to increased mitochondrial oxidative phosphorylation (OXPHOS), which is inconsistent with the decreased OXPHOS of highly-fused mitochondria observed in previous studies. In this paper, we have used the live-cell nanoscope to observe and quantify the structure of mitochondrial cristae, and the behavior of mitochondria and lysosomes in patient-derived SLC25A46 mutant fibroblasts. The results show that the cristae have been markedly damaged in the mutant fibroblasts, but there is no corresponding increase in mitophagy. This study suggests that severely damaged mitochondrial cristae might be the predominant cause of reduced OXPHOS in SLC25A46 mutant fibroblasts. This study demonstrates the utility of nanoscopebased imaging for realizing the sub-mitochondrial morphology, mitophagy and mitochondrial dynamics in living cells, which may be particularly valuable for the quick evaluation of pathogenesis of mitochondrial morphological abnormalities.
\end{abstract}

Keywords: Nanoscope, Mitochondrial disease, SLC25A46, Cristae, Mitophagy

\section{Background}

The mitochondrion is the cellular organelle which is critical for energy metabolism in mammals and most other eukaryotes. Mitochondrial dysfunction caused by nuclear DNA (nDNA) or mitochondrial DNA (mtDNA) defects lead to cellular respiratory chain and energy metabolism

\footnotetext{
*Correspondence: jiajie.diao@uc.edu; thuang29@buffalo.edu

'Weiwei Zou and Qixin Chen contributed equally to this study

${ }^{3}$ Department of Cancer Biology, University of Cincinnati College of Medicine, Cincinnati, OH 45267, USA

${ }^{5}$ Present Address: Department of Pediatrics, Jacobs School of Medicine and Biomedical Sciences, University at Buffalo, Buffalo, NY 14203, USA

Full list of author information is available at the end of the article
}

disorders, resulting in a group of multi-system diseases $[1,2]$. A number of mitochondrial diseases present aberrant mitochondrial morphology, including mitochondrial fragmentation or excessive mitochondrial fusion, which have an effect on mitochondrial function, leading to dysfunction of vital organs and tissues and accordingly threatening patients' health and survival [3-5].

We first identified $S L C 25 A 46$ as a pathogenic gene related to mitochondrial function [6]. SLC25A46 mutations can lead to highly fused mitochondria and decreased mitochondrial oxidative phosphorylation (OXPHOS). This result contradicts the traditional view that mitochondrial fusion is beneficial to the

(c) The Author(s) 2021. This article is licensed under a Creative Commons Attribution 4.0 International License, which permits use, sharing, adaptation, distribution and reproduction in any medium or format, as long as you give appropriate credit to the original author(s) and the source, provide a link to the Creative Commons licence, and indicate if changes were made. The images or other third party material in this article are included in the article's Creative Commons licence, unless indicated otherwise in a credit line to the material. If material is not included in the article's Creative Commons licence and your intended use is not permitted by statutory regulation or exceeds the permitted use, you will need to obtain permission directly from the copyright holder. To view a copy of this licence, visit http://creativeco mmons.org/licenses/by/4.0/. The Creative Commons Public Domain Dedication waiver (http://creativecommons.org/publicdomain/ zero/1.0/) applies to the data made available in this article, unless otherwise stated in a credit line to the data. 
improvement of OXPHOS [7]. Therefore, from the perspective of sub-mitochondrial structure and mitophagy, it is more likely that damage to the sub-mitochondrial structure or an increase in mitophagy underly the mitochondrial dysfunction in the patients with SLC25A46 mutations.

Over the past few decades, mitochondrial three-dimensional (3D) structure and the structure of mitochondrial cristae could only be observed by transmission electron microscopy (TEM). However, TEM cannot be applied to living cells and 3D observation, and is also time-consuming and expensive [8]. On the other hand, the confocal microscope is unable to visualize and quantitatively calculate the structure of sub-mitochondria [9]. 3D-structured illumination microscopy (SIM) is nanoscale microscopy which illuminates living cells by patterned excitation light, and then reconstructs the image in silico to achieve a doubling of the spatial resolution in all three dimensions [10]. With a spatial resolution of 100$120 \mathrm{~nm}, 3 \mathrm{D}$-SIM was recently developed to observe and quantify mitochondrial morphology, sub-mitochondrial structure, mitophagy, mitochondrial dynamics, and the interaction of organelles [11-13]. Therefore, 3D-SIM, can accomplish multiple experimental purposes with a single technique by achieving the comprehensive observation of mitochondrial 3D morphology, sub-mitochondrial structure, mitophagy, and mitochondrial dynamics. In this paper, we have set out to take advantage of the live-cell nanoscope-3D-SIM to dynamically observe the mitochondrial and sub-mitochondrial morphology in the fibroblasts derived from the patients carrying biallelic mutations in SLC25A46. The results were consistent with the TEM by another group [14], suggesting that our 3D-SIM based analysis technique was reliable. Combined with the sub-mitochondrial structure identification/ quantification and mitochondria-lysosome interaction quantification methods developed by our group [15-18], we found that the damage of mitochondrial cristae was the most probable cause of mitochondrial dysfunction in patients with $S L C 25 A 46$ mutations, and that the damaged mitochondrial cristae did not induce mitophagy. This study indicates 3D-SIM can be used to evaluate sub-mitochondrial structural damage in living cells and identify the pathology for patients with mitochondrial disease.

\section{Results}

\section{Patient-derived SLC25A46 mutant fibroblasts reveal} abnormal mitochondrial functions

Sanger sequencing results showed a homozygous, missense point mutation (c.1005 A $>$ T; p.Glu335Asp) in SLC25A46 mutant fibroblasts (Additional file 1). To examine mitochondrial function of SLC25A46 mutant fibroblasts, the mitochondrial respiration function was investigated by examining OCR under both basal conditions and drug-induced mitochondrial stress using the Seahorse assay. The OCR was found to be significantly decreased in patient-derived SLC25A46 mutant fibroblasts compared to normal fibroblasts (Fig. 1a). After a detailed analysis, the basal respiration, oxygen consumption for adenosine triphosphate (ATP) production, maximum oxygen consumption capacity of mitochondria, proton-leaked oxygen consumption, non-mitochondrial respiration, and the spare respiratory capacity in patientderived SLC25A46 mutant fibroblasts were all lower than that of normal fibroblasts (Fig. 1b). This result is consistent with the previous finding that SLC25A46 mutations cause decreased mitochondrial OXPHOS.

The 3-(4,5-dimethylthiazol-2-yl)-2,5-diphenyl tetrazolium bromide (MTT) assay reflects the metabolic ability of living cells by measuring the proliferation rates of cells. The results of this assay for the mutant and normal fibroblasts showed no apparent difference in the number of living cells between these two types of fibroblasts on day 2 after cell seeding. However, subsequent to this time point, the normal fibroblasts showed vigorous metabolism and rapid proliferation rate on day 4, day 6 , and day 8 (Fig. 1c). Thus, the metabolic ability and cell proliferation rate of $S L C 25 A 46$ mutant fibroblasts were significantly lower than that of normal fibroblasts (Fig. 1c). The imaging results showed that the cell density of normal fibroblasts was close to $80-90 \%$ on day 8 , while it only reached $40-50 \%$ in SLC25A46 mutant fibroblasts (Fig. 1d). Our results suggest that $S L C 25 A 46$ mutations affect cell proliferation, probably through decreased mitochondrial respiration.

\section{Nanoscope-3D-SIM imaging system demonstrates mitochondrial hyper-fusion in the living patient-derived SLC25A46 mutant fibroblasts}

The decreased metabolic ability of mutant fibroblasts suggested that the mitochondrial function in SLC25A46 mutant fibroblasts has been disturbed. To examine whether this mutation causes any changes in mitochondrial morphology, we used a nanoscope-3D-SIM imaging approach to observe mitochondrial morphology in these two human cell lines. The images showed that the normal fibroblasts had round or medium length mitochondria (Fig. 2a), while the SLC25A46 mutant fibroblasts showed slender, hyper-fused mitochondria (Fig. 2b). Imaris software (Nikon, Tokyo, Japan) was used to identify and analyze the mitochondrial morphology (Fig. 2c, d). The results showed that the number of mitochondria in SLC25A46 mutant fibroblasts was significantly lower than what was observed in normal fibroblasts. In contrast, the average area and volume of mitochondria 

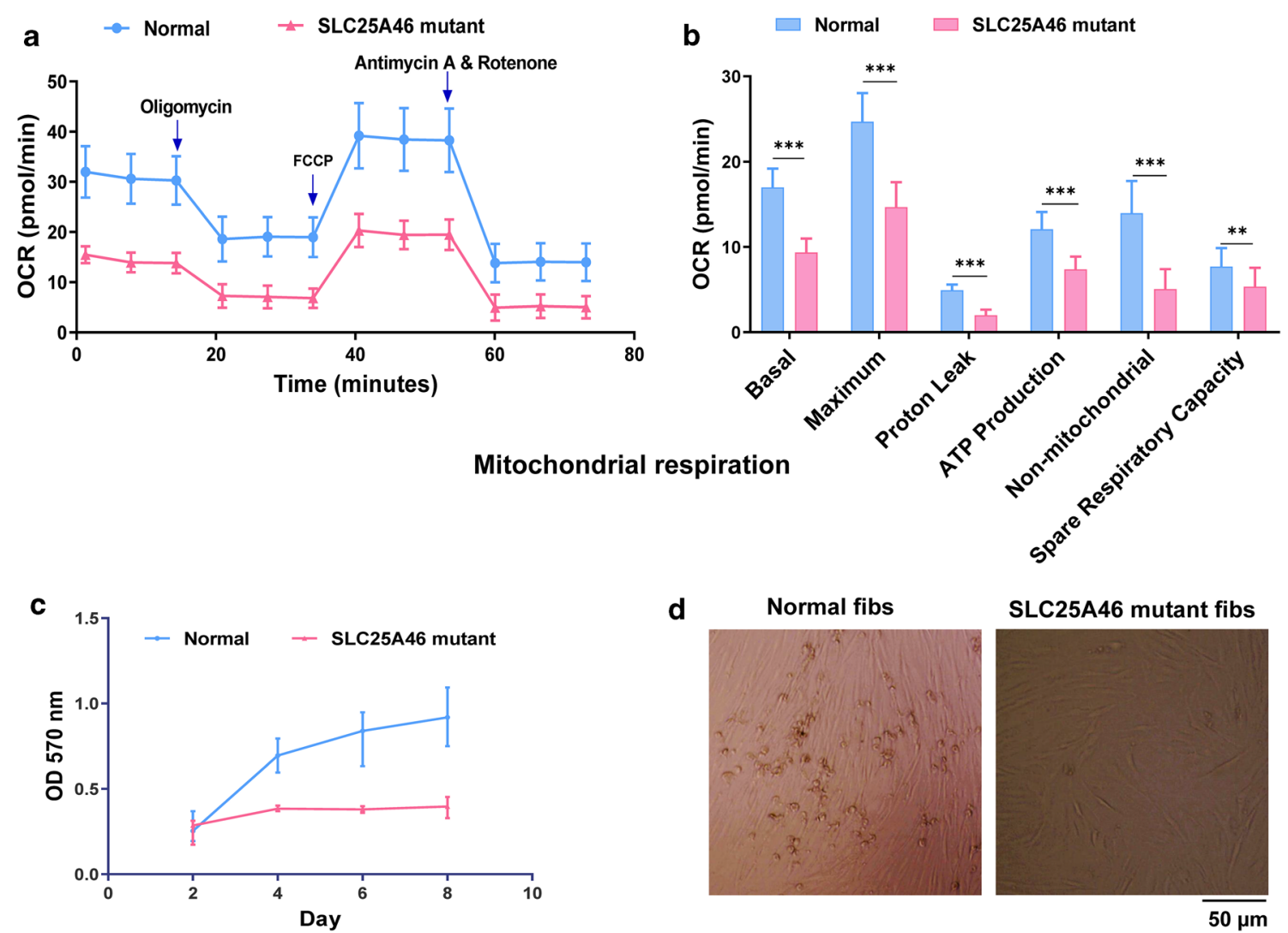

SLC25A46 mutant fibs

Fig. 1 Comparative analysis of mitochondrial respiration and cell proliferation in human-derived normal and SLC25A46 mutant fibroblasts. The oxygen consumption rate $(\mathrm{OCR})(\mathbf{a}, \mathbf{b})$ and the cell proliferation rate during one week after the seeding of the cells $(\mathbf{c}, \mathbf{d})$ are shown for human-derived normal and SLC25A46 mutant fibroblasts. Data appear as mean $\pm S D ;{ }^{* *} p<0.001,{ }^{* *} \mathrm{p}<0.01$, as compared to normal fibroblasts

in mutant cells were significantly greater than those in normal fibroblasts (Fig. 2e). The comparative analysis of mitochondrial morphology showed aberrant hyper fusion of mitochondria in the patient-derived SLC24A46 mutant fibroblasts.

\section{Severe damage of mitochondrial cristae in patient-derived SLC25A46 mutant fibroblasts}

Previously, mitochondrial fusion was considered to facilitate OXPHOS, and an increase of mitochondrial fusion was assumed to improve the mitochondrial OXPHOS level $[7,19]$. Mediated mitochondrial fusion was therefore regarded as a new therapeutic target for mitochondrial diseases [20, 21]. However, our group found that the highly-fused mitochondria from SLC25A46 mutant fibroblasts resulted in reduced OXPHOS [6] and our results here also confirmed that SLC25A46 mutant fibroblasts have a low respiration as measured by SeaHorse (Fig. 1). What is the underlying pathogenesis for SLC25A46 mutations? One possibility is that it is related to alterations in the cristae, one of the most critical structures of the inner mitochondrial membrane (IMM), which are deemed as the core of ATP production and mitochondrial respiratory function $[22,23]$. Therefore, we decided to investigate whether structural defects of mitochondrial cristae lead to decreased OXPHOS.

Using algorithm-based SIM imaging technology previously developed by our team [15], we first identified and extracted cristae, then quantitatively analyzed the mitochondrial cristae for human-derived normal and patientderived SLC25A46 mutant fibroblasts. The images showed that the mitochondrial cristae structure was visible and abundant in normal fibroblasts (Fig. 3a). In contrast, the cristae structure was damaged or even vanished in SLC25A46 mutant fibroblasts (Fig. 3b). After quantification analysis, the mean cristae number (Fig. 3c), cristae length (Fig. 3d), and cristae area (Fig. 3e) of SLC25A46 mutant fibroblasts all showed significantly lower values than those observed in normal fibroblasts.

\section{SLC25A46 mutations do not significantly alter mitophagy in fibroblasts}

Mitophagy is the general process by which the cell removes severely damaged mitochondria, consequently 

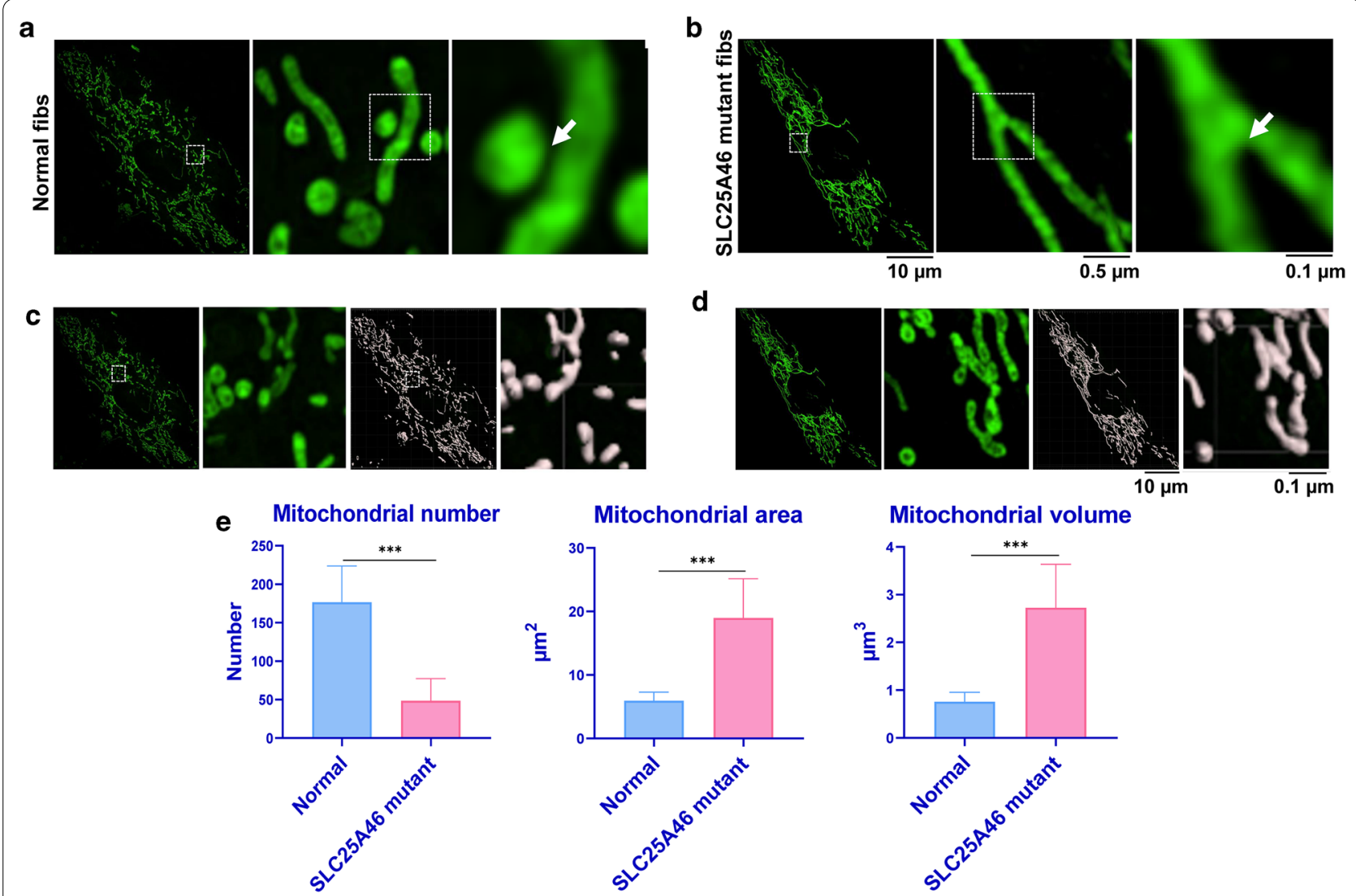

Mitochondrial volume

$0.1 \mu \mathrm{m}$

Fig. 2 Comparative analysis of mitochondrial morphology in human-derived normal and SLC25A46 mutant fibroblasts. The mitochondrial morphology is shown for human-derived normal (a) and SLC25A46 mutant fibroblasts (b). Quantitative analysis of mitochondrial number was performed by Imaris (c, d). The results of the quantitative analysis of the mitochondrial area and volume by the Imaris software are also shown (e). Data are presented as mean $\pm S D(n=8) ;{ }^{* * *} p<0.001$, as compared to normal fibroblasts

achieving the purpose of "quality control" of mitochondria within living cells $[24,25]$. We observed highly-fused mitochondria with severely damaged cristae structures in SLC25A46 mutant fibroblasts. This raised the obvious question of whether or not these abnormal mitochondria induce mitophagy? Using the SIM image-based mitochondria-lysosome co-location analysis method in living cells [16], we can observe and quantify mitophagy in normal and SLC25A46 mutant fibroblasts (Fig. 4a-c).

Our results demonstrate that only a low level of mitophagy occurs in both normal and mutant cell lines (Fig. 4a, b). After quantitative analysis, there was no statistically significant difference in the value of mitochondrial-lysosome co-location between the normal and SLC25A46 mutant fibroblasts (Fig. 4c). Western Blot also confirmed that the values of the light chain 3-II (LC3II)-II/LC3-I ratio were comparable between normal and SLC25A46 mutant fibroblasts (Fig. 4d), which was consistent with the results of the SIM image-based analysis method. This result is confirmed in our 3D-SIM analysis by straightforwardly monitoring the mitochondrial dynamics and the mitochondria-lysosome interaction dynamics in human fibroblasts (Fig. 4e).

Our results showed that a combination of nanoscope with a quantification analysis strategy can not only be used to observe mitochondrial morphology, but also to detect and quantify damage to sub-mitochondrial structures, assess the extent of mitophagy, and monitor the dynamics of mitochondria and lysosomes (Fig. 5). This provides a novel means of observing and identifying pathology in patients with mitochondrial disease, which is critical to guide the development of the treatment.

\section{Discussion}

SLC25A46 is responsible for encoding a mitochondrial solute carrier protein [26]. We previously identified SLC25A46 is the human homolog of Ugo1, a protein of Saccharomyces cerevisiae and located in the mitochondrial outer membrane and involved in mitochondrial fusion $[6,27,28]$. So far, SLC25A46 has been found to be associated with various human diseases. Homozygous or compound heterozygous mutations of SLC25A46 led to 


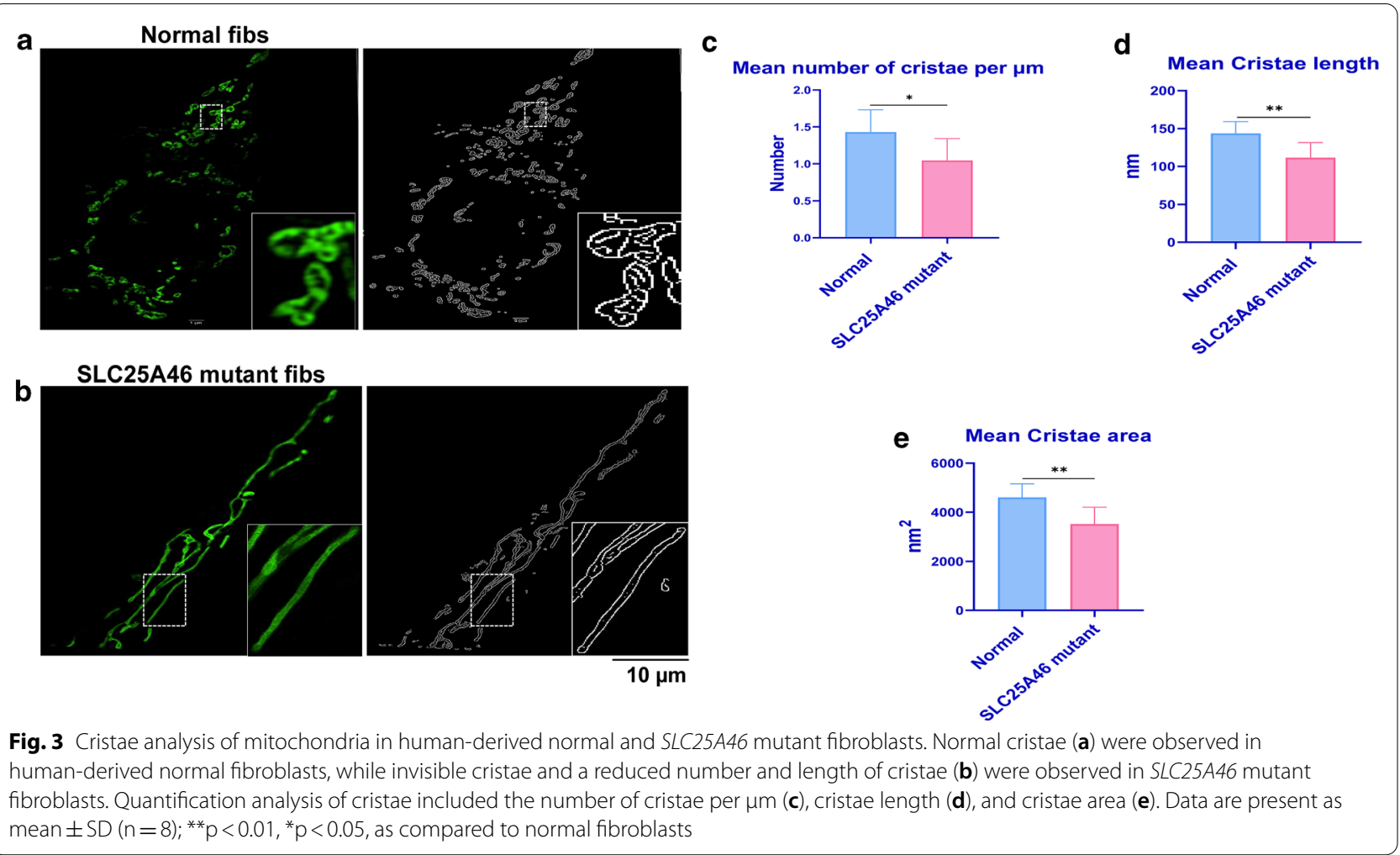

a range of clinical syndromes, with the clinical feature of optic atrophy, cerebellar atrophy, progressive myoclonic ataxia, axonal peripheral neuropathy, autosomal recessive cerebellar ataxias (ARCA), lethal congenital pontocerebellar hypoplasia, and even Parkinson's disease [6, 26, 29-34]. Mice with Slc25a46 dysfunction developed severe motor impairment, optic atrophy, and developmental defects of the nervous system, as well as premature death [35-37].

Currently, SLC25A46 is believed to affect mitochondrial dynamics due to the interaction with dominant optic atrophy 1 (OPA1) and Mitofusin-2 (MFN2) [14, 38]. The hyper-fused mitochondria and reduced mitochondrial respiratory function presented in patient-derived SLC25A46 mutant fibroblasts have also been confirmed by this study, as well as previous studies, which were supposed to be the pathogenic mechanism of a series of neurological diseases [39]. The MTT assay results from this study also strengthened the idea that the metabolic capacity of SLC25A46 mutant fibroblasts is significantly lower than that of control cells. However, there exists a contradiction about the morphology of highly-fused mitochondria and the decline of mitochondrial function. Traditionally, mitochondrial fusion has been verified to be vital for maintaining mtDNA stability and improving the tolerance of cells to high mtDNA mutations $[40,41]$. At the same time, mitochondrial fusion is also a protective factor for maintaining normal mitochondrial respiration function. The absence of mitochondrial fusion in the cerebellum has also been shown to result in a malformed mitochondrial distribution and function [42]. Moreover, mitochondrial fusion is required to support the normal development of embryos [3].

Why then do the SLC25A46 mutant cells examined in our study show mitochondrial hyper-fusion but a decrease in mitochondrial respiratory function? The respiratory function of mitochondria is a series of oxidation-reduction reactions mediated by multiple complexes located on the mitochondrial inner cristae, which eventually produce ATP and provide energy for the tissues and cells in living organisms [43, 44]. From this viewpoint, we hypothesized that SLC25A46 mutation causes structural abnormalities of cristae in highly fused mitochondria, consequently affecting mitochondrial respiratory function. Based on the identification and quantification method of mitochondrial cristae developed by our group, we analyzed the mitochondrial internal cristae of patient-derived SLC25A46 mutant fibroblasts and human-derived normal fibroblasts. Our results showed that, compared with normal mitochondria, the number of mitochondrial cristae decreased, the length of cristae shortened, and the area of cristae was reduced in the SLC25A46 mutant fibroblasts. We even observed the disappearance of cristae in some mitochondria. Therefore, 


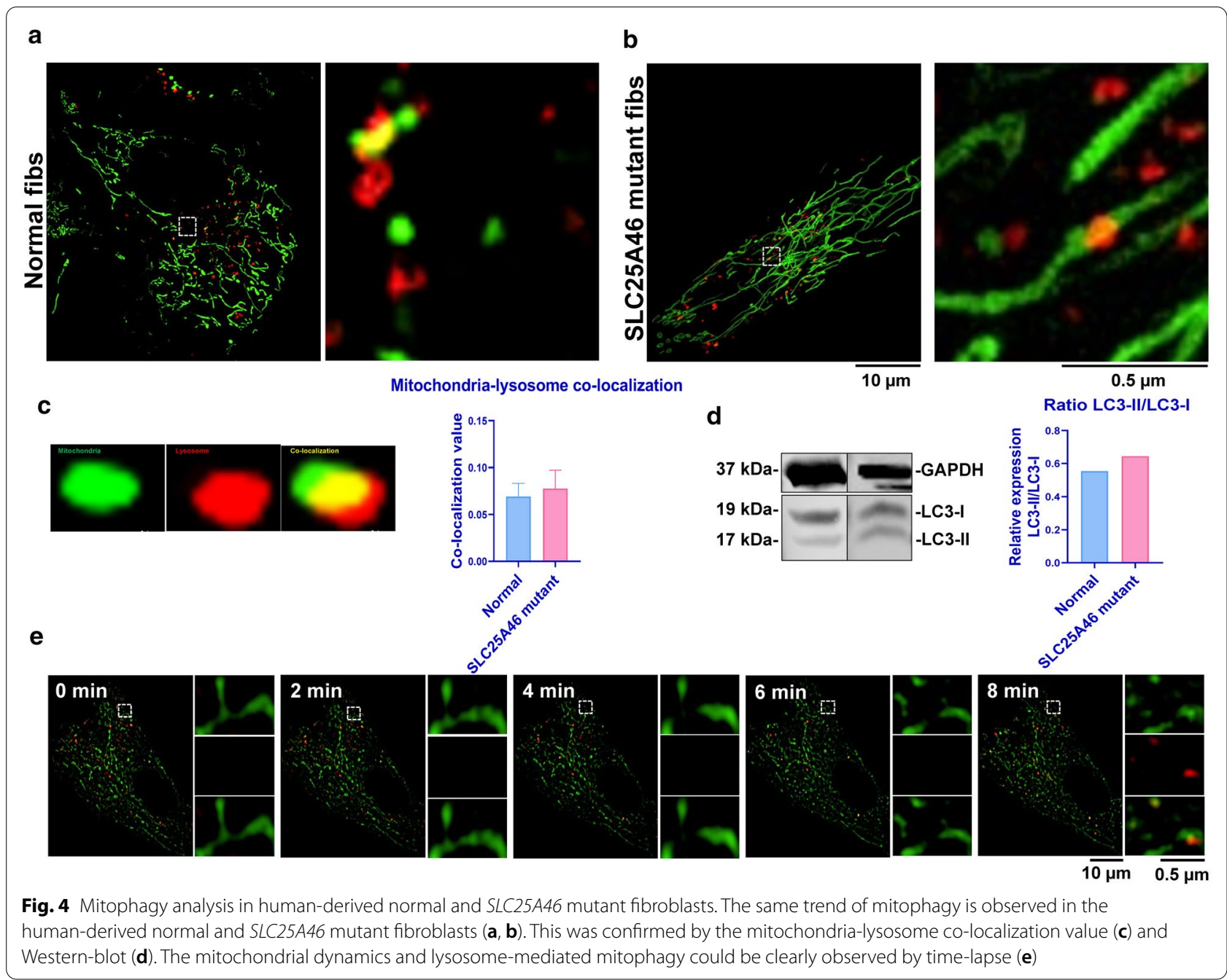

we posited that the structure of mitochondrial cristae in the mutant cells was damaged, which therefore affected the mitochondrial respiratory function, as reflected by decreased aerobic respiration, reduced ATP generation, and decreased metabolic capacity. Researchers have suggested that SLC25A46 plays a vital role in the interaction between the major structural proteins of the mitochondrial outer membrane and the mitochondrial cristae, and it is crucial for maintaining the structure and stability of the mitochondrial cristae. Immunoblot analysis revealed that MICOS complex subunit 60 (MIC60) and MICOS complex subunit 19 (MIC19) - two critical proteins of mitochondrial contact site and cristae organizing system (MICOS) complex-were clearly decreased in patient-derived SLC25A46 mutant fibroblasts. An immunoprecipitation experiment showed that SLC25A46 co-immunoprecipitated with MIC60, MIC19, OPA1 (located on IMM), MFN1 and MFN2 (located on OMM) [14]. MICOS complex, especially MIC60 and MIC19, is a crucial factor in cristae biogenesis [45, 46]. Therefore, SLC25A46 is believed to be not only involved in maintaining the stability of OMM, but also an essential protein in the interaction and communication between the OMM and IMM, as well as the formation and maintenance of mitochondrial cristae [14]. Our group as well as others have used TEM and observed the significantly decreased cristae number and length from patientderived mitochondria [14]. Our results in 3D SIM are consistent with the findings from TEM analysis, verifying the reliability of our nanoscope-based method.

Mitophagy is known to be an autophagy process that selectively eliminates excess or damaged mitochondria. It plays a vital role in regulating the number of mitochondria in cells and maintaining mitochondrial quality control. It is involved in many physiological and pathological processes $[47,48]$. Severe injury of mitochondrial cristae can induce mitophagy as well $[49,50]$. Therefore, we also hypothesized that the damaged mitochondrial 


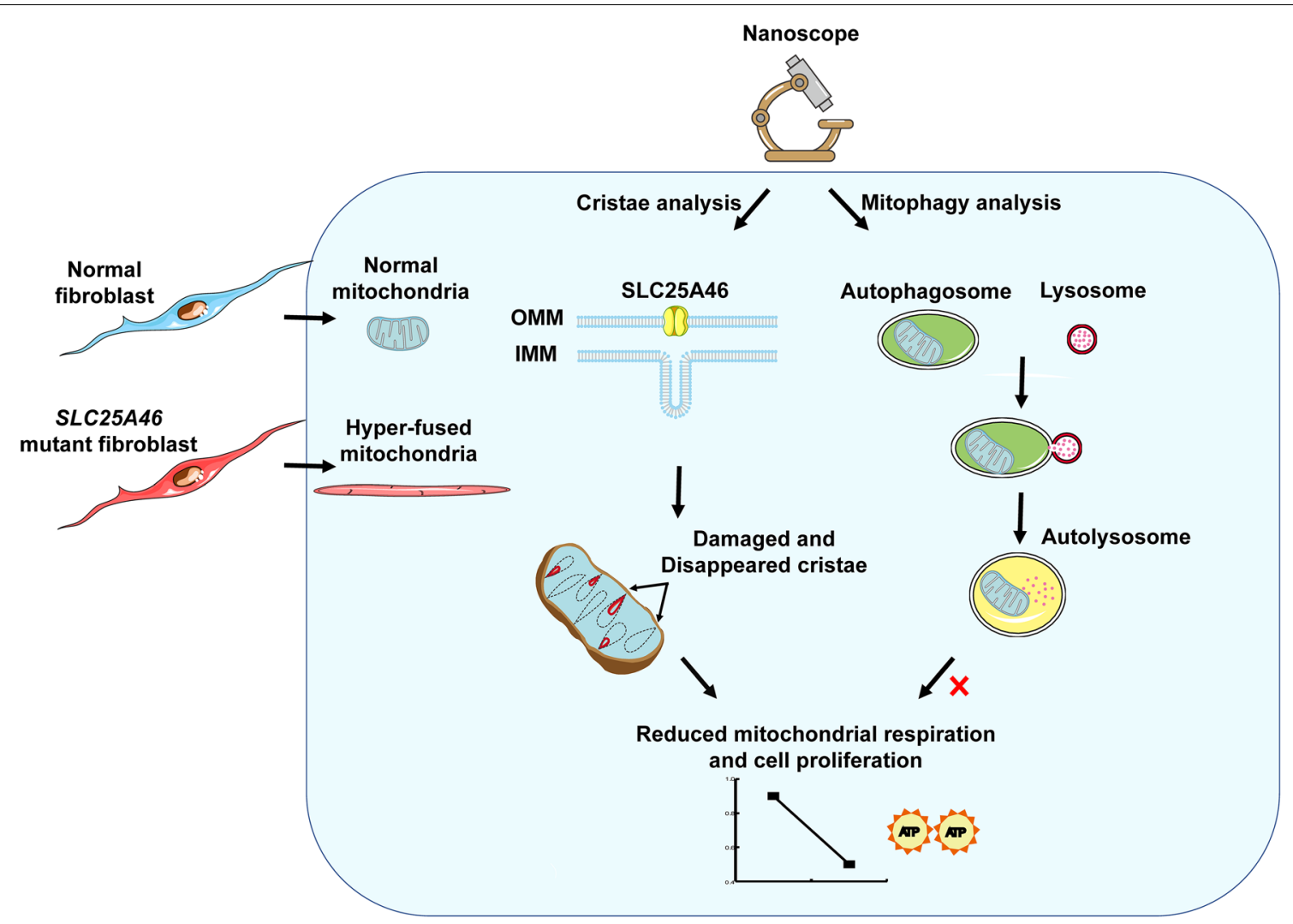

Fig. 5 Summary of results for the examination of mitochondrial dysfunction using the nanoscope. The nanoscope can be used to closely analyze the structure of mitochondrial cristae, mitophagy, and mitochondrial dynamics in living cells, which is an extremely suitable application for the clinical analysis of the causes of mitochondrial dysfunction in patients with mitochondrial diseases

cristae would increase the rate of mitophagy in SLC25A46 mutant fibroblasts. We monitored the dynamic changes of mitochondrial and lysosomal behavior in SLC25A46 mutant fibroblasts in real-time. We observed a contact and co-localization phenomenon between lysosomes and mitochondria after mitochondrial fragmentation in SLC25A46 mutant fibroblasts. However, using the SIM image-based mitophagy quantification method, we determined that the overall tendency of mitophagy in the SLC25A46 mutant fibroblasts was not statistically different from that in normal fibroblasts, although mitophagy did occur in some mitochondria in the SLC25A46 mutant fibroblasts. Consequently, although the mitochondrial cristae were severely damaged in the SLC25A46 mutant fibroblasts, the damaged cristae alone did not appear to induce mitophagy in this particular cell line. Why then did the damage to mitochondrial cristae not stimulate mitophagy? Is it because the mitochondrial cristae damage alone is not enough to trigger mitophagy, or are there defects in the mitophagy process in SLC25A46 mutant fibroblasts? According to the characteristics of the mitophagy process, the precondition for mitophagy is the membrane permeability change after mitochondrial damage. This leads to mitochondrial depolarization and induces the activation of mitophagy-related proteins. Subsequently, damaged mitochondria are wrapped by the early autophagosomes, and then mitophagosomes are formed. Therefore, we will likely focus on the following questions in the near future: (1) Is there any change in mitochondrial membrane potential in SLC25A46 mutant fibroblasts? We can thus determine whether mitochondrial damage is sufficient to induce mitophagy. (2) Is there any alteration in the expression of mitophagyrelated proteins (such as PINK1, Parkin, MUL1, etc.) in SLC25A46 mutant fibroblasts? We can also explore this question by studying whether there are mitochondrial autophagy deficiencies in the mutant fibroblasts. Currently, no studies have reported the mitophagy status of SLC25A46 mutant cells.

\section{Conclusions}

Overall, this study shows that nanoscope-based imaging is a reliable method for analyzing the sub-mitochondrial morphology, mitophagy and mitochondrial dynamics in 
living cells. This method may be particularly valuable for the quick evaluation of the pathogenesis of mitochondrial morphological abnormalities. Utilizing this tool, we were able to show that severely damaged mitochondrial cristae may be the predominant cause of reduced mitochondrial respiratory dysfunction in SLC25A46 mutant fibroblasts. This approach also showed that damaged mitochondrial cristae do not appear to be sufficient to induce a significant increase in mitophagy in this particular condition.

The significance of this study is that is will likely be possible to apply this method to a wide range of mitochondrial diseases by examining the sub-mitochondrial structure of living cells. Moreover, the 3D-SIM can image up to 4 fluorecent channels, which can also be used for simultaneously monitoring the dynamics of fluorophorelabeled proteins. Another advantage of this method is that we are able to identify the mitochondrial 3D morphology, mitophagy, and mitochondrial dynamics using one technique. Therefore, it is exceptionally suited for those patients with mitochondrial diseases related to morphological disruption. The operation of the device is simple and rapid, which is valuable for the quick assessment of mitochondrial morphological abnormalities and the identification of the potential pathology, and may eventually help facilitate the screening of lead compounds for the treatment of such disorders.

\section{Methods}

\section{Cell culture}

The human-derived normal fibroblasts and patientderived SLC25A46 mutant (c.1005 A>T, p.Glu335Asp) fibroblasts cell lines were acquired after informed consent was obtained from the patients. The cells were cultured in Dulbecco's modified Eagle's medium (DMEM) medium (Gibco, Thermo Fisher Scientific, USA) with 10\% Fetal Bovine Serum (FBS) (Gibco, Thermo Fisher Scientific, USA) and 100 units/ml Anti-Anti (containing streptomycin and penicillin) (Gibco, Thermo Fisher Scientific, USA) and incubated in a $5 \% \mathrm{CO}_{2}, 37^{\circ} \mathrm{C}$ and $100 \%$ humidity incubator.

\section{Nanoscope-3D-SIM imaging}

The cells were seeded in a glass-bottom culture dish (MatTek Life Sciences, USA) and cultured for $24 \mathrm{~h}$ in 2 $\mathrm{ml}$ DMEM containing 10\% FBS and 100 units/ml AntiAnti. Before imaging, cells were first washed three times with a pre-warmed DMEM medium and then were incubated in a DMEM medium containing $100 \mathrm{nM}$ MitoTracker Green (Invitrogen, USA) for half an hour. Cells for mitophagy analysis were co-incubated in DMEM medium containing $100 \mathrm{nM}$ Mito-Tracker Green (Invitrogen, USA) and Lyso-Tracker Red (Invitrogen, USA) for half an hour. Cells were then washed three times with
DMEM. The stained cells were photographed using the 3D-structure illumination microscope (Nikon, Tokyo, Japan).

\section{Western blot}

Protease inhibitor cocktail (Sigma, USA) and $2 \times$ RIPA lysis and extraction buffer (Thermofisher Scientific, USA) were added to the centrifuged cell pellets, and were then sonicated for $5 \mathrm{~min}$ each time, three times in total. The protein concentration was measured using the Pierce BCA Protein Assay Kit (Thermofisher Scientific, USA). $30 \mu \mathrm{g}$ protein for each sample and $4 \times$ NuPAGE LDS Sample Buffer (Thermofisher Scientific, USA) were mixed at 4:1 ratio and denatured at $95{ }^{\circ} \mathrm{C}$ for $5 \mathrm{~min}$, and then separated in $4-12 \%$ Bis-Tris gel (Invitrogen, USA). The gel was transferred onto a PVDF membrane (Invitrogen, USA) through the iBlot 2 gel transfer device (Life Technologies, USA). The transferred PVDF membrane was placed in the Intercept Blocking Buffer (LI-COR Biosciences, USA) for $45 \mathrm{~min}$, and then incubated overnight in the primary antibody, rabbit anti-LC3B (cell signaling technology, USA) diluted in the blocking buffer at a ratio of 1:200 with Tween 20 diluted in the blocking buffer at a ratio of 1:1000. Rabbit anti-GAPDH (cell signaling technology, USA) was also diluted in the blocking buffer at a ratio of 1:2000 and set as the loading control. The next day, the PVDF membrane was washed for $10 \mathrm{~min}$ each time, three times in total. Then, the membrane was incubated in the secondary antibody, IRDye $800 \mathrm{CW}$ Goat anti-Rabbit IgG (LI-COR Biosciences, USA), for $120 \mathrm{~min}$. The bands were detected by the LI-COR Odyssey Clx Imaging System (LI-COR Biosciences, Lincoln, NE).

\section{Sanger sequencing for mutation detection}

To detect the point mutation of SLC25A46 in humanderived normal and patient-derived fibroblasts, genomic DNA was extracted using DNeasy Blood \& Tissue Kit (Qiagen, USA). PCR products of $186 \mathrm{bp}$ in length were amplified using GoTag master mixes (Promega, USA). The following primer set was used for the amplification: Forward: TGCCAGTCTTTGTTCTGACG and Reverse: CCAAACACTCCTTCCTCCTG. The reactions were performed following the thermal cycling program: $95{ }^{\circ} \mathrm{C}$ for $2 \mathrm{~min}$, followed by 30 cycles of $95^{\circ} \mathrm{C}$ for $30 \mathrm{~s}, 56^{\circ} \mathrm{C}$ for $30 \mathrm{~s}$, and $72{ }^{\circ} \mathrm{C}$ for $30 \mathrm{~s}$. A final extension step was then performed at $72{ }^{\circ} \mathrm{C}$ for $4 \mathrm{~min}$.

\section{Oxygen consumption rate (OCR) measurement}

Human-derived normal and patient-derived SLC25A46 mutant cells were seeded at a density of $1.0 \times 10^{4}$ cells/ well with DMEM supplemented with 10\% FBS in XFe96 cell culture plates (Agilent Technologies, USA). After incubation for $24 \mathrm{~h}$, the DMEM medium was removed 
and changed with the warmed XF DMEM Medium supplemented with $1 \mathrm{mM}$ sodium pyruvate, $10 \mathrm{mM}$ glucose and $2 \mathrm{mM} \mathrm{L}$-glutamine at $\mathrm{pH}$ 7.4. All cells were treated with $1 \mu \mathrm{M}$ oligomycin A, $1 \mu \mathrm{M}$ FCCP, and $500 \mathrm{nM}$ rotenone/antimycin A. The OCRs of the cells was assessed by using the XF Cell Mito Stress Test Kit (Agilent Technologies, USA). The Seahorse XF96 analyzer (Agilent Technologies, USA) was used for OCR measurement.

\section{Cell proliferation rate measurement (MTT assay)}

Human-derived normal and patient-derived SLC25A46 mutant fibroblasts were seeded in 96 well plates (Corning, USA) at a density of $3.0 \times 10^{3}$ cells/well with DMEM supplemented with $10 \% \mathrm{FBS}$ and incubated at $37^{\circ} \mathrm{C}, 5 \%$ $\mathrm{CO}_{2} .10 \mu \mathrm{l}$ of MTT solution (Roche, USA) was added to $100 \mathrm{ul}$ culture medium in each well at a final concentration of $0.5 \mathrm{mg} / \mathrm{ml}$. The following process was implemented according to the manual provided by the kit. The absorbance was detected at $570 \mathrm{~nm}$ by the microplate reader (BioTek, USA).

\section{Statistical analysis}

Graphpad Prism 7 software was used to display data. Independent-samples $T$-test was used for statistical analysis. " was defined as $P<0.05$, ** as $P<0.01$, *** as $P<0.001$, and ${ }^{* * * * *}$ as $P<0.0001$.

\section{Abbreviations}

OXPHOS: Oxidative phosphorylation; nDNA: Nuclear DNA; mtDNA: Mitochondrial DNA; 3D: Three dimensional; SIM: Structured illumination microscopy; ATP: Adenosine triphosphate; MTT: 3-(4,5-Dimethylthiazol-2-yl)-2,5-diphenyl tetrazolium bromide; LC3: Light chain 3; OCR: Oxygen consumption rate; IMM: Inner mitochondrial membrane; OMM: Outer mitochondrial membrane; ARCA : Autosomal recessive cerebellar ataxias; OPA1: Dominant optic atrophy 1; MFN2: Mitofusin-2; MIC60: MICOS complex subunit 60; MIC19: MICOS complex subunit 19; MICOS: Mitochondrial contact site and cristae organizing system;
\end{abstract} DMEM: Dulbecco's modified Eagle's medium; FBS: Fetal bovine serum.

\section{Supplementary Information}

The online version contains supplementary material available at https://doi. org/10.1186/s12951-021-00882-9.

Additional file 1. Sanger sequencing of the mutant variant of humanderived normal and SLC25A46 mutant fibroblasts. Sanger sequencing results showed a homozygous, missense point mutation (c.1005A>T; p.Glu335Asp) in SLC25A46 mutant fibroblasts (B). The normal variant at this site is also shown for comparison (A).

\section{Acknowledgements}

Not applicable.

\section{Authors' contributions}

TH and JJ conceived the idea and designed the experiments. WZ, QC, YL and $X L$ carried out the experiments of the manuscript. WZ, QC, JS, and JD participated in the analysis and interpretation of the data. WZ prepared the figures and the original draft. QC, YL, and XL corrected the figures. TH, JJ, and JS reviewed and revised the manuscript. All authors read and approved the final manuscript.

\section{Funding}

This work was supported by the Hadley J. Foundation and the National Institute of Child Health \& Development 1R01HD092989.

\section{Availability of data and materials}

The datasets used and/or analysed during the current study are available from the corresponding author on reasonable request.

\section{Declarations}

\section{Ethics approval and consent to participate}

Informed consent was obtained from the patients before taking the samples. This study was approved by the Institutional Review Board of Cincinnati Children's Hospital Medical Center.

\section{Consent for publication}

Not applicable.

\section{Competing interests}

The authors declare that they have no competing interests.

\section{Author details}

${ }^{1}$ Division of Human Genetics, Cincinnati Children's Hospital Medical Center, 3333 Burnet Avenue, Cincinnati, OH 45229, USA. ² Department of Obstetrics and Gynecology, Reproductive Medicine Center, The First Affiliated Hospital of Anhui Medical University, Hefei 230022, China. ${ }^{3}$ Department of Cancer Biology, University of Cincinnati College of Medicine, Cincinnati, OH 45267, USA. ${ }^{4}$ Institute of Materia Medica, Shandong First Medical University \& Shandong Academy of Medical Sciences, Jinan 250062, China. ${ }^{5}$ Present Address: Department of Pediatrics, Jacobs School of Medicine and Biomedical Sciences, University at Buffalo, Buffalo, NY 14203, USA. ${ }^{6}$ Department of Pediatrics, Xiangya Hospital, Central South University, Changsha 410008, Hunan, China. ${ }^{7}$ School of Laboratory Medicine and Life sciences, Wenzhou Medical University, Wenzhou 325035, Zhejiang, China.

Received: 4 November 2020 Accepted: 5 May 2021

Published online: 13 May 2021

\section{References}

1. Craven L, Alston CL, Taylor RW, Turnbull DM. Recent advances in mitochondrial disease. Annu Rev Genom Hum Genet. 2017;18:257-75.

2. Alston CL, Rocha MC, Lax NZ, Turnbull DM, Taylor RW. The genetics and pathology of mitochondrial disease. J Pathol. 2017;241(2):236-50.

3. Chen H, Detmer SA, Ewald AJ, Griffin EE, Fraser SE, Chan DC. Mitofusins Mfn 1 and Mfn2 coordinately regulate mitochondrial fusion and are essential for embryonic development. J Cell Biol. 2003;160(2):189-200.

4. Reddy PH. Inhibitors of mitochondrial fission as a therapeutic strategy for diseases with oxidative stress and mitochondrial dysfunction. J Alzheimer's Dis. 2014;40(2):245-56.

5. Chan DC. Mitochondrial dynamics and its involvement in disease. Annu Rev Pathol. 2020:15:235-59.

6. Abrams AJ, Hufnagel RB, Rebelo A, Zanna C, Patel N, Gonzalez MA, et al. Mutations in SLC25A46, encoding a UGO1-like protein, cause an optic atrophy spectrum disorder. Nat Genet. 2015;47(8):926-32.

7. Bonnay F, Veloso A, Steinmann V, Köcher T, Abdusselamoglu MD, Bajaj S, et al. Oxidative metabolism drives immortalization of neural stem cells during tumorigenesis. Cell. 2020;182(6):1490.e19-1507.e19.

8. Hu C, Shu L, Huang X, Yu J, Li L, Gong L, et al. OPA1 and MICOS regulate mitochondrial crista dynamics and formation. Cell Death Dis. 2020;11(10):940.

9. Fang H, Yao S, Chen Q, Liu C, Cai Y, Geng S, et al. De novo-designed nearinfrared nanoaggregates for super-resolution monitoring of lysosomes in cells, in whole organoids, and in vivo. ACS Nano. 2019;13(12):14426-36.

10. Hirano Y, Matsuda A, Hiraoka Y. Recent advancements in structuredillumination microscopy toward live-cell imaging. Microscopy. 2015:64(4):237-49.

11. Fang $H$, Geng $S$, Hao M, Chen Q, Liu M, Liu C, et al. Simultaneous $\mathrm{Zn}(2+)$ tracking in multiple organelles using super-resolution 
morphology-correlated organelle identification in living cells. Nat Commun. 2021;12(1):109.

12. Chen Q, Fang H, Shao X, Tian Z, Geng S, Zhang Y, et al. A dual-labeling probe to track functional mitochondria-lysosome interactions in live cells. Nat Commun. 2020;11(1):6290.

13. Qiu K, Du Y, Liu J, Guan JL, Chao H, Diao J. Super-resolution observation of lysosomal dynamics with fluorescent gold nanoparticles. Theranostics. 2020;10(13):6072-81.

14. Janer A, Prudent J, Paupe V, Fahiminiya S, Majewski J, Sgarioto N, et al. SLC25A46 is required for mitochondrial lipid homeostasis and cristae maintenance and is responsible for Leigh syndrome. EMBO Mol Med. 2016;8(9):1019-38.

15. Shao X, Chen Q, Hu L, Tian Z, Liu L, Liu F, et al. Super-resolution quantification of nanoscale damage to mitochondria in live cells. Nano Res. 2020;13(8):2149-55.

16. Chen Q, Shao X, Tian Z, Chen Y, Mondal P, Liu F, et al. Nanoscale monitoring of mitochondria and lysosome interactions for drug screening and discovery. Nano Res. 2019;12(5):1009-15.

17. Chen Q, Shao X, Hao M, Fang H, Guan R, Tian Z, et al. Quantitative analysis of interactive behavior of mitochondria and lysosomes using structured illumination microscopy. Biomaterials. 2020;250:120059.

18. Chen Q, Jin C, Shao X, Guan R, Tian Z, Wang C, et al. Super-resolution tracking of mitochondrial dynamics with an iridium(III) luminophore. Small. 2018;14(41):e1802166.

19. Yao CH, Wang R, Wang Y, Kung CP, Weber JD, Patti GJ. Mitochondrial fusion supports increased oxidative phosphorylation during cell proliferation. eLife. 2019;8:e41351.

20. Qi X, Qvit N, Su YC, Mochly-Rosen D. A novel Drp1 inhibitor diminishes aberrant mitochondrial fission and neurotoxicity. J Cell Sci. 2013;126(Pt 3):789-802.

21. Szabo A, Sumegi K, Fekete K, Hocsak E, Debreceni B, Setalo G Jr, et al. Activation of mitochondrial fusion provides a new treatment for mitochondria-related diseases. Biochem Pharmacol. 2018;150:86-96.

22. Baker N, Patel J, Khacho M. Linking mitochondrial dynamics, cristae remodeling and supercomplex formation: how mitochondrial structure can regulate bioenergetics. Mitochondrion. 2019;49:259-68.

23. Quintana-Cabrera R, Mehrotra A, Rigoni G, Soriano ME. Who and how in the regulation of mitochondrial cristae shape and function. Biochem Biophys Res Commun. 2018;500(1):94-101.

24. Pickles S, Vigié P, Youle RJ. Mitophagy and quality control mechanisms in mitochondrial maintenance. Curr Biol. 2018;28(4):R170-85.

25. Kiriyama Y, Nochi H. Intra- and intercellular quality control mechanisms of mitochondria. Cells. 2017;7(1):1.

26. Bitetto G, Malaguti MC, Ceravolo R, Monfrini E, Straniero L, Morini A, et al. SLC25A46 mutations in patients with Parkinson's disease and optic atrophy. Parkinsonism Relat Disord. 2020;74:1-5.

27. Sesaki H, Jensen RE. UGO1 encodes an outer membrane protein required for mitochondrial fusion. J Cell Biol. 2001;152(6):1123-34.

28. Coonrod EM, Karren MA, Shaw JM. Ugo1p is a multipass transmembrane protein with a single carrier domain required for mitochondrial fusion. Traffic. 2007;8(5):500-11.

29. Hammer MB, Ding J, Mochel F, Eleuch-Fayache G, Charles P, Coutelier M, et al. SLC25A46 mutations associated with autosomal recessive cerebellar ataxia in North African families. Neuro-degener Dis. 2017;17(4-5):208-12.

30. Wan J, Steffen J, Yourshaw M, Mamsa H, Andersen E, Rudnik-Schöneborn $\mathrm{S}$, et al. Loss of function of SLC25A46 causes lethal congenital pontocerebellar hypoplasia. Brain. 2016;139(11):2877-90.

31. Nguyen M, Boesten I, Hellebrekers DM, Mulder-den Hartog NM, de Coo IF, Smeets $\mathrm{HJ}$, et al. Novel pathogenic SLC25A46 splice-site mutation causes an optic atrophy spectrum disorder. Clin Genet. 2017;91(1):121-5.

32. Braunisch MC, Gallwitz H, Abicht A, Diebold I, Holinski-Feder E, Van Maldergem $L$, et al. Extension of the phenotype of biallelic loss-of-function mutations in SLC25A46 to the severe form of pontocerebellar hypoplasia type I. Clin Genet. 2018;93(2):255-65.

33. Charlesworth G, Balint B, Mencacci NE, Carr L, Wood NW, Bhatia KP. SLC25A46 mutations underlie progressive myoclonic ataxia with optic atrophy and neuropathy. Mov Disord. 2016;31(8):1249-51.

34. van DijkT, Rudnik-Schöneborn S, Senderek J, Hajmousa G, Mei H, Dusl M, et al. Pontocerebellar hypoplasia with spinal muscular atrophy (PCH1): identification of SLC25A46 mutations in the original Dutch PCH1 family. Brain. 2017;140(8):e46.

35. Li Z, Peng Y, Hufnagel RB, Hu YC, Zhao C, Queme LF, et al. Loss of SLC25A46 causes neurodegeneration by affecting mitochondrial dynamics and energy production in mice. Hum Mol Genet. 2017;26(19):3776-91.

36. Yang L, Slone J, Li Z, Lou X, Hu YC, Queme LF, et al. Systemic administration of AAV-SIc25a46 mitigates mitochondrial neuropathy in Slc25a46-/- mice. Hum Mol Genet. 2020;29(4):649-61.

37. Terzenidou ME, Segklia A, Kano T, Papastefanaki F, Karakostas A, Charalambous M, et al. Novel insights into SLC25A46-related pathologies in a genetic mouse model. PLoS Genet. 2017;13(4):e1006656.

38. Steffen J, Vashisht AA, Wan J, Jen JC, Claypool SM, Wohlschlegel JA, et al. Rapid degradation of mutant SLC25A46 by the ubiquitin-proteasome system results in MFN1/2-mediated hyperfusion of mitochondria. Mol Biol Cell. 2017;28(5):600-12.

39. Abrams AJ, Fontanesi F, Tan NBL, Buglo E, Campeanu IJ, Rebelo AP, et al. Insights into the genotype-phenotype correlation and molecular function of SLC25A46. Hum Mutat. 2018;39(12):1995-2007.

40. Chen $H$, Vermulst M, Wang YE, Chomyn A, Prolla TA, MCCaffery JM, et al. Mitochondrial fusion is required for mtDNA stability in skeletal muscle and tolerance of mtDNA mutations. Cell. 2010;141(2):280-9.

41. Sprenger $H G$, Langer $T$. The good and the bad of mitochondrial breakups. Trends Cell Biol. 2019;29(11):888-900.

42. Chen H, McCaffery JM, Chan DC. Mitochondrial fusion protects against neurodegeneration in the cerebellum. Cell. 2007;130(3):548-62.

43. Stoldt S, Wenzel D, Kehrein K, Riedel D, Ott M, Jakobs S. Spatial orchestration of mitochondrial translation and OXPHOS complex assembly. Nat Cell Biol. 2018;20(5):528-34.

44. Kühlbrandt W. Structure and function of mitochondrial membrane protein complexes. BMC Biol. 2015;13:89.

45. Stephan T, Brüser C, Deckers M, Steyer AM, Balzarotti F, Barbot M, et al. MICOS assembly controls mitochondrial inner membrane remodeling and crista junction redistribution to mediate cristae formation. EMBO J. 2020;39(14):e104105.

46. Harner M, Körner C, Walther D, Mokranjac D, Kaesmacher J, Welsch U, et al. The mitochondrial contact site complex, a determinant of mitochondrial architecture. EMBO J. 2011;30(21):4356-70.

47. Ashrafi G, Schwarz TL. The pathways of mitophagy for quality control and clearance of mitochondria. Cell Death Differ. 2013;20(1):31-42.

48. Hamacher-Brady A, Brady NR. Mitophagy programs: mechanisms and physiological implications of mitochondrial targeting by autophagy. Cell Mol Life Sci. 2016;73(4):775-95.

49. Hytti M, Korhonen E, Hyttinen JMT, Roehrich $H$, Kaarniranta K, Ferrington DA, et al. Antimycin A-induced mitochondrial damage causes human RPE cell death despite activation of autophagy. Oxid Med Cell Longev. 2019. https://doi.org/10.1155/2019/1583656.

50. Wang H, Zhang T, Ge X, Chen J, Zhao Y, Fu J. Parkin overexpression attenuates $A \beta$-induced mitochondrial dysfunction in HEK293 cells by restoring impaired mitophagy. Life Sci. 2020;244:117322.

\section{Publisher's Note}

Springer Nature remains neutral with regard to jurisdictional claims in published maps and institutional affiliations. 